\title{
Development of a Potent and Selective HDAC8 Inhibitor
}

Oscar J. Ingham, Ronald M. Paranal, William B. Smith, Randolph A. Escobar, Han Yueh, Tracy Snyder, John A. Porco, Jr., James E. Bradner, Aaron B. Beeler

General Information: ${ }^{1} \mathrm{H}$ NMR spectra were recorded at $500 \mathrm{MHz}$ at ambient temperature with $\mathrm{CDCl}_{3}$ as a solvent unless otherwise stated. ${ }^{3} \mathrm{C}$ NMR spectra were recorded at $126 \mathrm{MHz}$ at ambient temperature with $\mathrm{CDCl}_{3}$ as a solvent unless otherwise stated. Chemical shifts are reported in parts per million relative to $\mathrm{CDCl}_{3}\left({ }^{1} \mathrm{H}, \delta 7.26 ;{ }^{13} \mathrm{C}, \delta 77.0\right)$. Data for ${ }^{1} \mathrm{H}$ NMR are reported as follows: chemical shift, multiplicity, integration $(\mathrm{app}=$ apparent, par obsc= partially obscure, ovrlp= overlapping, $\mathrm{s}=$ singlet, $\mathrm{d}=$ doublet, $\mathrm{t}=$ triplet, q quartet, $\mathrm{m}=$ multiplet) and coupling constants are reported as values in hertz. All ${ }^{13} \mathrm{C}$ NMR spectra were recorded with complete proton decoupling. High-resolution mass spectra were obtained on a Waters QTof API US. Flash chromatography was performed using Silicycle UltraPure silica gel (particle size 40-63 $\mu \mathrm{m}$ ). 


\section{Synthesis and Characterization Data}

(S)-methyl 2-(5-iodo-4-phenyl-1H-1,2,3-triazol-1-yl)-3-phenylpropanoate (29).<smiles>COC(=O)[C@H](Cc1ccccc1)n1nnc(-c2ccccc2)c1I</smiles>

(lodoethynyl)benzene ( $2.78 \mathrm{~g}, 12.2 \mathrm{mmol}, 1.5$ eq.) (freshly-prepared according to the procedure Fokin and coworkers ${ }^{1}$ ) was dissolved in $20 \mathrm{~mL}$ of anhydrous THF and transferred to a flame-dried flask containing $154 \mathrm{mg}(0.81 \mathrm{mmol}, 10 \mathrm{~mol} \%)$ of Copper (I) lodide. A solution of (S)-methyl 2-azido-3-phenylpropanoate $(1.67 \mathrm{~g}, 8.13$ mmol, 1 eq.) in $20 \mathrm{~mL}$ of anhydrous THF was added to the flask via syringe. $2.27 \mathrm{~mL}$ of triethylamine ( $16.26 \mathrm{mmol}, 2$ eq.) was added to the flask via syringe and the resulting solution was stirred overnight at room temperature. The yellow solution was filtered through Celite and concentrated in vacuo. The crude material was purified by flash chromatography $\left(\mathrm{SiO}_{2}, 20 \%\right.$ EtOAc/Hexanes) to afford $3.14 \mathrm{~g}(89 \%)$ of the desired iodide as a white solid. ${ }^{1} \mathrm{H} \mathrm{NMR}\left(500 \mathrm{MHz}, \mathrm{CDCl}_{3}\right) 3.79(5 \mathrm{H}, \mathrm{m}), 5.49(1 \mathrm{H}, \mathrm{dd}, J=6.4,9.3 \mathrm{~Hz}), 7.10(2 \mathrm{H}, \mathrm{dd}, J=1.7,7.6 \mathrm{~Hz}), 7.19$ $7.24(3 \mathrm{H}, \mathrm{m}), 7.39(1 \mathrm{H}, \mathrm{t}, J=7.3 \mathrm{~Hz}), 7.45(2 \mathrm{H}, \mathrm{t}, J=6.8 \mathrm{~Hz}), 7.90(2 \mathrm{H}, \mathrm{d}, J=6.8 \mathrm{~Hz}) ;{ }^{13} \mathrm{C} \mathrm{NMR}(126 \mathrm{MHz}$, $\mathrm{CDCl}_{3}$ ) 37.30, 53.14, 64.23, 78.78, 127.19, 127.40, 128.35, 128.49, 128.55, 128.95, 129.91, 135.53, 149.12, 167.73; HRMS calculated for $\mathrm{C}_{18} \mathrm{H}_{17} \mathrm{IN}_{3} \mathrm{O}_{2}^{+}(\mathrm{M}+\mathrm{H}): 434.0360$, found: 434.0378 .

(S)-methyl 2-(5-(cyclopropylethynyl)-4-phenyl-1H-1,2,3-triazol-1-yl)-3-phenylpropanoate (30).

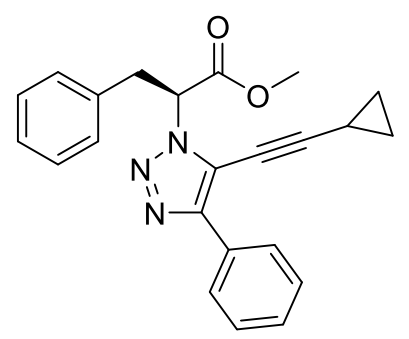

Into a flame-dried flask was added $288 \mathrm{mg}(0.41 \mathrm{mmol}, 10 \mathrm{~mol} \%)$ of bis(triphenylphosphine)palladium(II) dichloride and $78 \mathrm{mg}(0.41 \mathrm{mmol}, 10 \mathrm{~mol}$ \%) of copper (I) iodide To the flask was added $1.79 \mathrm{~g}$ ( $4.1 \mathrm{mmol}, 1$ eq.) of iodotriazole (29) and anhydrous toluene $(40 \mathrm{~mL}, 0.1 \mathrm{M})$. Into the solution was added $1.74 \mathrm{~mL}$ of cyclopropylacetylene $(20.5 \mathrm{mmol}, 5 \mathrm{eq}$.) via syringe, followed by $1.14 \mathrm{~mL}$ of triethylamine $(8.2 \mathrm{mmol}, 2$ eq.). The resulting yellow solution was stirred at $80^{\circ} \mathrm{C}$ for 5 hours. The solution was filtered through Celite and concentrated in vacuo. The black residue was suspended in ethyl acetate and extracted 3 times with water and brine. The organic layer was dried over sodium sulfate and concentrated in vacuo. The crude material was purified by flash chromatography $\left(\mathrm{SiO}_{2}, 17 \% \mathrm{EtOAc} /\right.$ Hexanes) to yield $1.20 \mathrm{~g}(79 \%)$ of the desired alkyne as a yellow oil. ${ }^{1} \mathrm{H}$ NMR $\left(500 \mathrm{MHz}, \mathrm{CDCl}_{3}\right) 0.85(2 \mathrm{H}, \mathrm{m}), 0.99(2 \mathrm{H}, \mathrm{m}), 1.55(1 \mathrm{H}, \mathrm{m}), 3.72(2 \mathrm{H}$, m), $3.78(3 \mathrm{H}, \mathrm{s}), 5.49(1 \mathrm{H}, \mathrm{dd}, J=5.4,9.8 \mathrm{~Hz}), 7.10(2 \mathrm{H}, \mathrm{dd}, J=2.0,7.8 \mathrm{~Hz}), 7.19-7.24(3 \mathrm{H}, \mathrm{m}), 7.35(1 \mathrm{H}, \mathrm{t}$, $J=7.3 \mathrm{~Hz}), 7.42(2 \mathrm{H}, \mathrm{t}, J=7.6 \mathrm{~Hz}), 8.10(2 \mathrm{H}, \mathrm{dd}, J=1.0,8.3 \mathrm{~Hz}) ;{ }^{13} \mathrm{C} \mathrm{NMR}\left(106 \mathrm{MHz}, \mathrm{CDCl}_{3}\right) 0.42,9.10,36.89$, 53.03, 61.43, 62.83, 108.08, 118.33, 125.90, 127.11, 128.32, 128.46, 128.56, 128.90, 130.22, 135.68, 146.90, 168.17; HRMS calculated for $\mathrm{C}_{23} \mathrm{H}_{22} \mathrm{~N}_{3} \mathrm{O}_{2}{ }^{+}(\mathrm{M}+\mathrm{H})$ : 372.1707, found: 372.1712 .

\footnotetext{
${ }^{1}$ Hein J. E., Tripp J. C., Krasnova L. B., Sharpless K. B., Fokin V. V. Angew. Chem. Int. Ed. 2009, 48, 8018-8021.
} 
(S)-3-phenyl-2-(4-phenyl-5-(phenylethynyl)-1H-1,2,3-triazol-1-yl)propanoic acid (11).

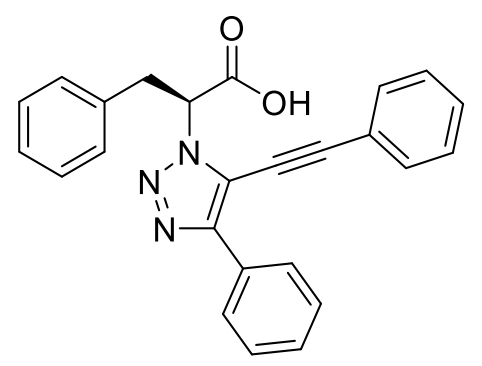

$122 \mathrm{mg}$ of ester ( $0.3 \mathrm{mmol}, 1$ eq.) was added to a glass vial equipped with a magnetic stir bar and $14 \mathrm{mg}(0.6 \mathrm{mmol}, 2$ eq.) of $\mathrm{LiOH}$ was added. The mixture was dissolved in $3.0 \mathrm{~mL}(0.1 \mathrm{M})$ of THF/MeOH (4:1) and stirred for 1 hour at room temperature. The solution was acidified with $1 \mathrm{~N} \mathrm{HCl}$ to $\mathrm{pH}$ $=2$ and extracted with ethyl acetate. The organic layer was dried over sodium sulfate and concentrated in vacuo. The crude material was purified by flash chromatography $\left(\mathrm{SiO}_{2}, 5 \% \mathrm{MeOH}\right.$ in $\left.\mathrm{DCM}\right)$ to afford 110 $\mathrm{mg}(93 \%)$ of the desired compound as a white solid. ${ }^{1} \mathrm{H}$ NMR $\left(500 \mathrm{MHz}, \mathrm{CD}_{3} \mathrm{OD}\right) 3.78(2 \mathrm{H}, \mathrm{m}), 5.61(1 \mathrm{H}, \mathrm{d}$, $J=9.3 \mathrm{~Hz}), 7.11(5 \mathrm{H}, \mathrm{m}), 7.37(6 \mathrm{H}, \mathrm{m}), 7.52(2 \mathrm{H}, \mathrm{m}), 8.00(2 \mathrm{H}, \mathrm{d}, J=7.3 \mathrm{~Hz}) ;{ }^{13} \mathrm{C} N M R\left(126 \mathrm{MHz}, \mathrm{CD}_{3} \mathrm{OD}\right)$ 38.53, 76.17, 103.69, 119.87, 122.50, 127.24, 127.99, 129.62, 129.94, 129.97, 130.02, 131.10, 131.26, 132.67, 138.56, 148.44, 173.31 (br, weak); HRMS calculated for $\mathrm{C}_{25} \mathrm{H}_{20} \mathrm{~N}_{3} \mathrm{O}_{2}{ }^{+}(\mathrm{M}+\mathrm{H})$ : 394.1556 , found: 394.1560 .

\section{S)-N-hydroxy-3-phenyl-2-(4-phenyl-5-(phenylethynyl)-1H-1,2,3-triazol-1-yl)propanamide (4).}

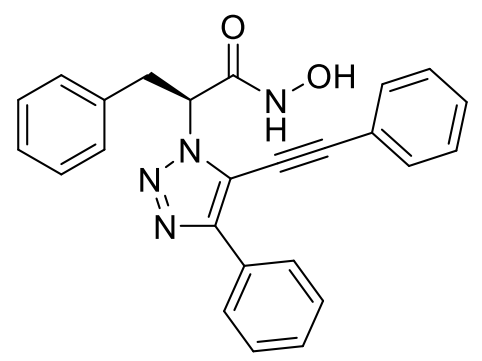

Into a flask was added $7.4 \mathrm{mg}(0.15 \mathrm{mmol}, 0.5$ eq.) of sodium cyanide and $1.5 \mathrm{~mL}$ of methanol. A solution of $122 \mathrm{mg}$ of ester in $1.5 \mathrm{~mL}$ THF was added via syringe. Into the solution was added $184 \mu \mathrm{L}$ of $50 \%$ aqueous hydroxylamine and the yellow reaction was stirred 16 hours at room temperature. The solution was diluted with water and extracted with EtOAc. The organic layer was dried over sodium sulfate and concentrated in vacuo. The resulting residue was purified by flash chromatography $\left(\mathrm{SiO}_{2}, 5 \% \mathrm{MeOH}\right.$ in $\left.\mathrm{DCM}\right)$ to afford $76 \mathrm{mg}(62 \%)$ of the desired compound as a white solid. ${ }^{1} \mathrm{H}$ NMR (500 $\mathrm{MHz}$, acetone- $\left.\mathrm{D}_{6}\right) 2.96(1 \mathrm{H}, \mathrm{br}),. 3.73-3.79(2 \mathrm{H}, \mathrm{m}), 5.74(1 \mathrm{H}, \mathrm{t}, J=7.5 \mathrm{~Hz}), 7.15(1 \mathrm{H}, \mathrm{t}, J=7.1 \mathrm{~Hz}), 7.21$ $(2 \mathrm{H}, \mathrm{t}, J=7.3 \mathrm{~Hz}), 7.26(2 \mathrm{H}, \mathrm{d}, J=7.3 \mathrm{~Hz}), 7.39(1 \mathrm{H}, \mathrm{t}, J=7.3 \mathrm{~Hz}), 7.47-7.51(5 \mathrm{H}, \mathrm{m}), 7.68(2 \mathrm{H}, \mathrm{d}, J=7.8$ $\mathrm{Hz}), 8.16(2 \mathrm{H}, \mathrm{d}, J=7.3 \mathrm{~Hz}) ;{ }^{13} \mathrm{C}$ NMR $\left(126 \mathrm{MHz}\right.$, acetone- $\left.\mathrm{D}_{6}\right) 37.79,63.40,75.90,103.71,118.61,122.27$, $126.81,127.84,129.30,129.51,129.70,130.14,130.80,131.34,132.57,137.29,147.87$, 164.97; HRMS calculated for $\mathrm{C}_{25} \mathrm{H}_{21} \mathrm{~N}_{4} \mathrm{O}_{2}{ }^{+}(\mathrm{M}+\mathrm{H}): 409.1665$, found: 409.1657 .

\section{(S)-2-(5-(cyclopropylethynyl)-4-phenyl-1H-1,2,3-triazol-1-yl)-N-hydroxy-3-phenylpropanamide (11).}

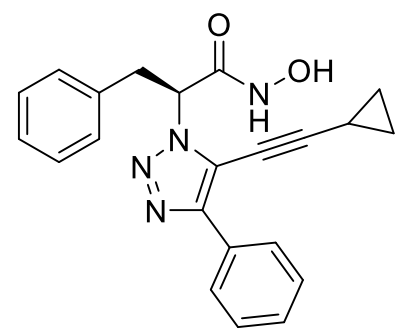

Into a flash was added $18.0 \mathrm{mg}(0.36 \mathrm{mmol}, 0.5 \mathrm{eq}$.) of sodium cyanide was and $3.5 \mathrm{~mL}$ of $\mathrm{MeOH}$. A solution of $265 \mathrm{mg}$ of the ester in $3.5 \mathrm{~mL} \mathrm{THF}$ was then added via syringe. To the reaction was added $435 \mu \mathrm{L}$ of $50 \%$ aqueous hydroxylamine and the yellow solution was stirred 16 hours at room temperature. The solution was diluted with water and extracted with EtOAc. The organic layer dried over sodium sulfate and concentrated in vacuo. The resulting residue was purified by flash chromatography $\left(\mathrm{SiO}_{2} 5 \% \mathrm{MeOH} /\right.$ DCM) to afford $173 \mathrm{mg}(65 \%)$ of the desired compound as a white solid. ${ }^{1} \mathrm{H}$ NMR (500 MHz, $\mathrm{d}_{4}-\mathrm{MeOD}$ ) $0.80(2 \mathrm{H}, \mathrm{m}), 0.98(2 \mathrm{H}, \mathrm{m}), 1.47(1 \mathrm{H}, \mathrm{m}), 3.51(1 \mathrm{H}, \mathrm{dd}, J=11.2,14.2 \mathrm{~Hz}), 3.71(1 \mathrm{H}, \mathrm{dd}, J=3.9,14.2 \mathrm{~Hz}), 5.49$ 
$(1 \mathrm{H}, \mathrm{dd}, J=3.9,11.2 \mathrm{~Hz}), 6.96(2 \mathrm{H}, \mathrm{m}), 7.17-7.20(3 \mathrm{H}, \mathrm{m}), 7.37(1 \mathrm{H}, \mathrm{t}, J=7.3 \mathrm{~Hz}), 7.43(2 \mathrm{H}, \mathrm{t}, J=7.3 \mathrm{~Hz})$, $7.99(2 \mathrm{H}, \mathrm{d}, J=8.8 \mathrm{~Hz}) ;{ }^{13} \mathrm{C} \mathrm{NMR}\left(100 \mathrm{MHz}, \mathrm{d}_{4}-\mathrm{MeOD}\right)$ 0.02, 8.55, 37.07, 60.83, 62.59, 109.09, 118.98, $125.9,127.16,128.55,128.65,128.71,129.16,130.07$, 136.09, 147.10, 165.20; HRMS calculated for $\mathrm{C}_{22} \mathrm{H}_{21} \mathrm{~N}_{4} \mathrm{O}_{2}{ }^{+}(\mathrm{M}+\mathrm{H}): 373.1659$, found: 373.1665 . 
II. NMR Spectra


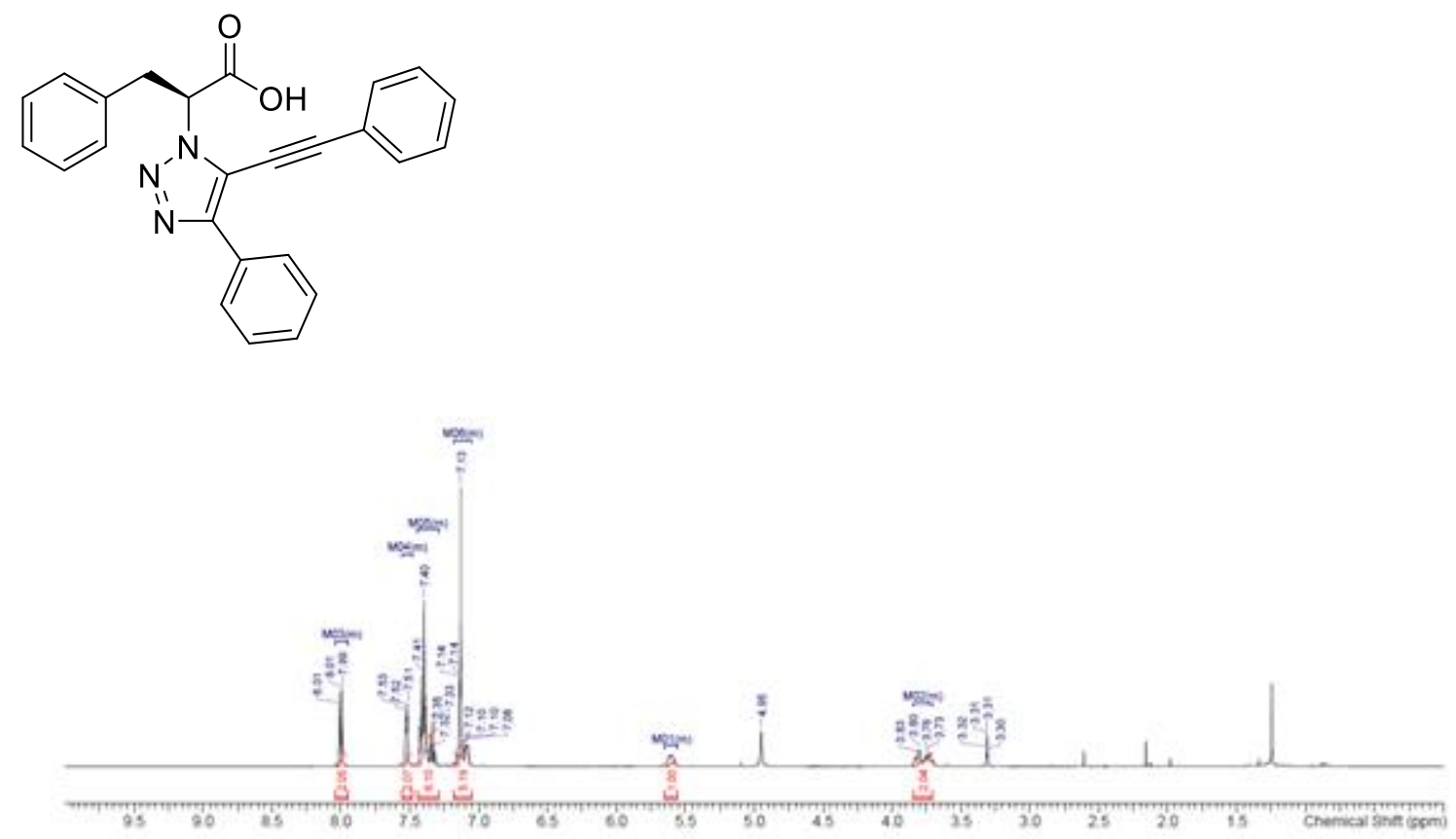

HY-29-COOH-Ph-C2_CARBON_01

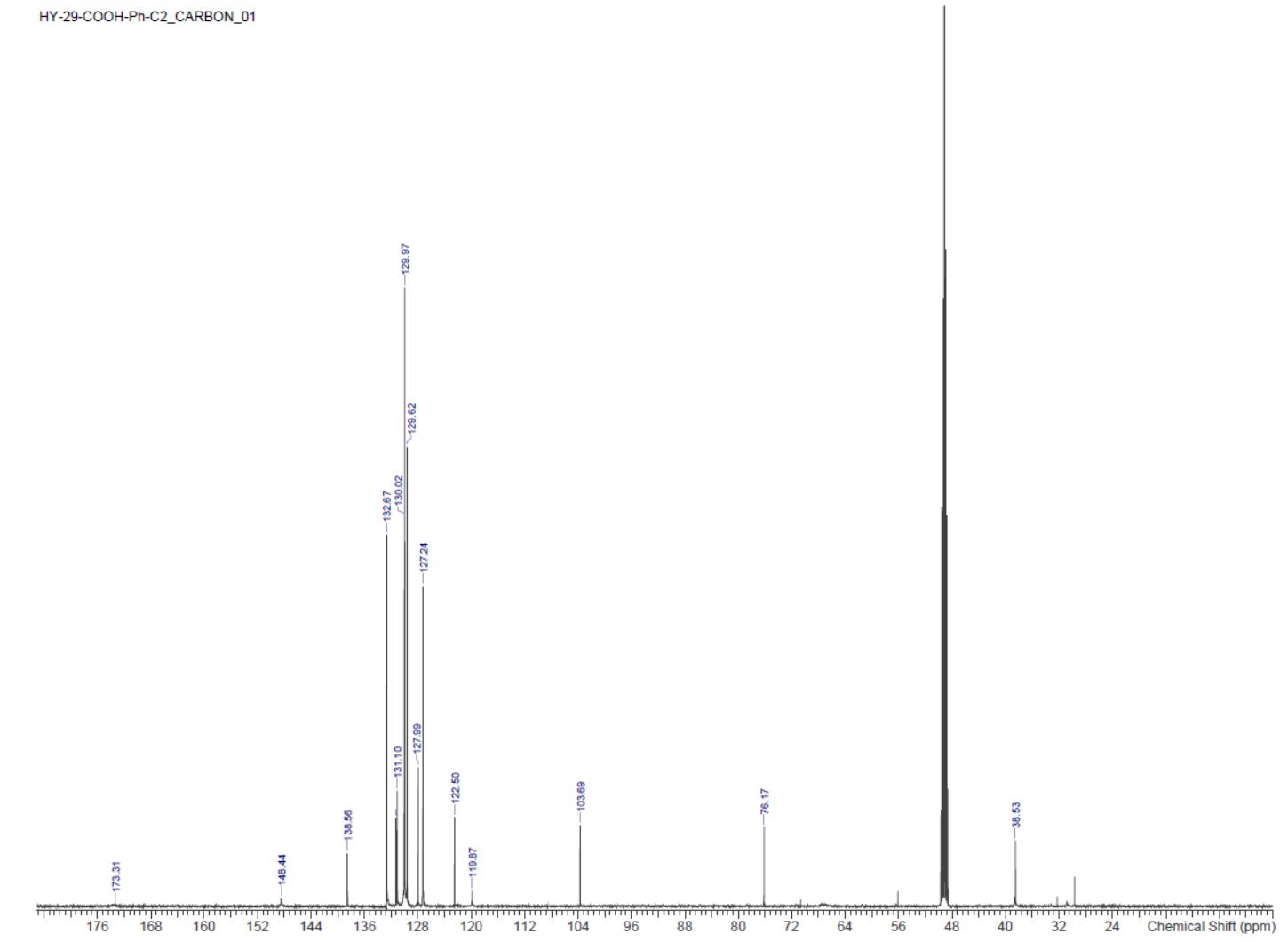





HY-29-03_CARBON_03
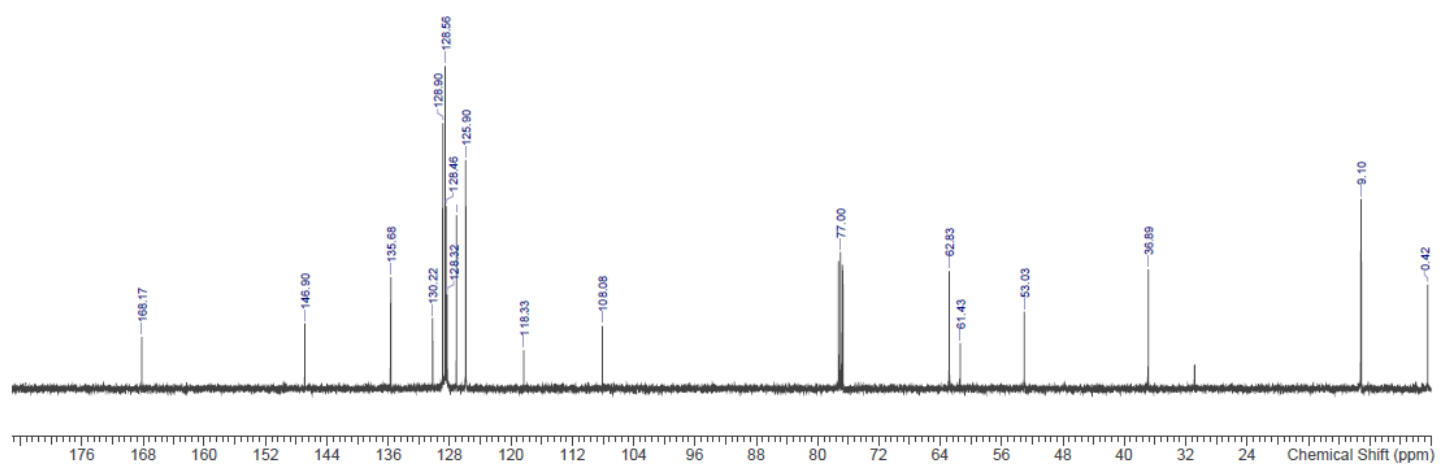




HY-29-04Ph-C_CARBON_01





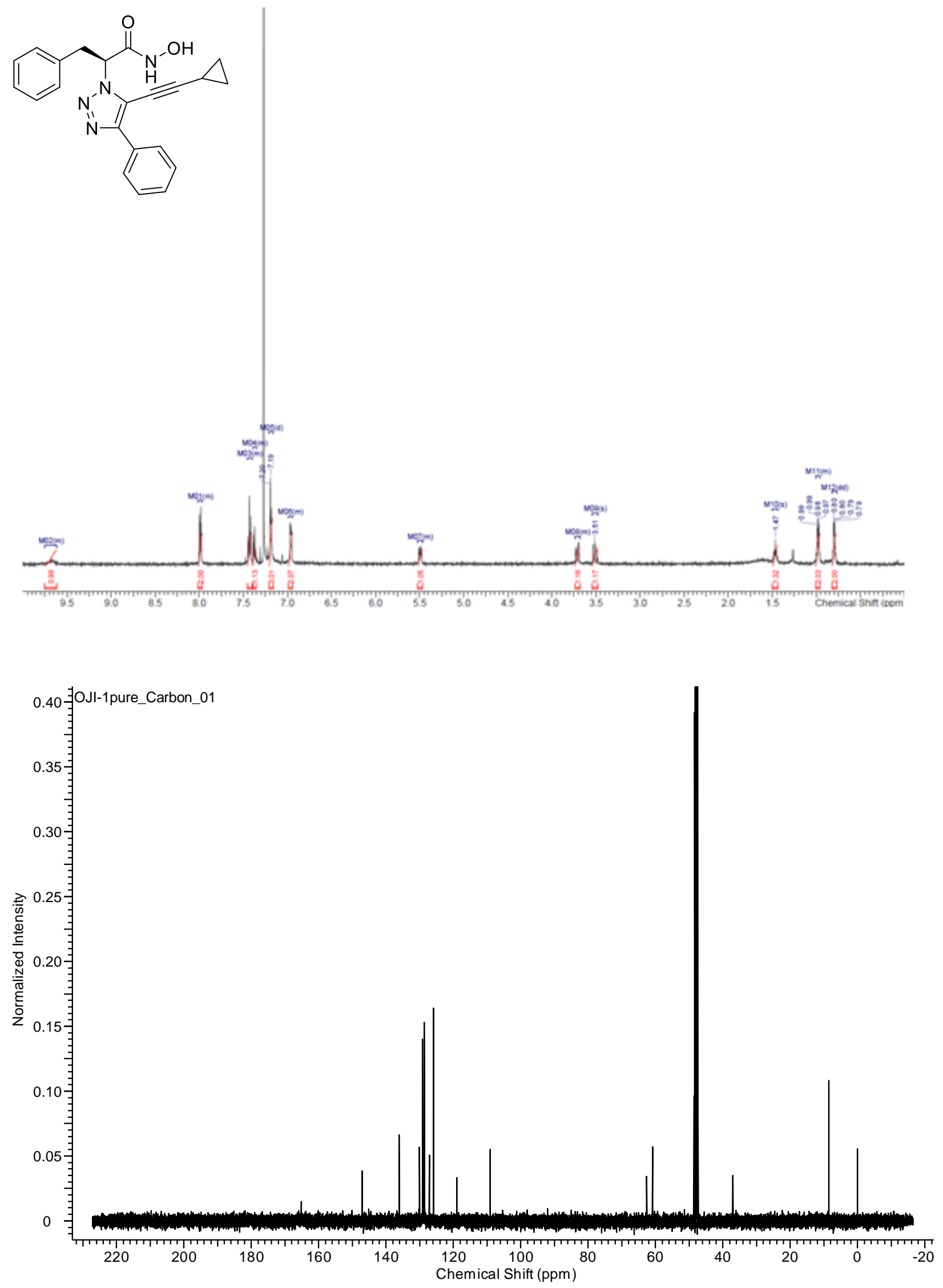


\section{HDAC Inhibition Procedure and Data}

General Procedure for HDAC Inhibition Assay: HDAC assays were carried out as described previously ${ }^{2,3}$
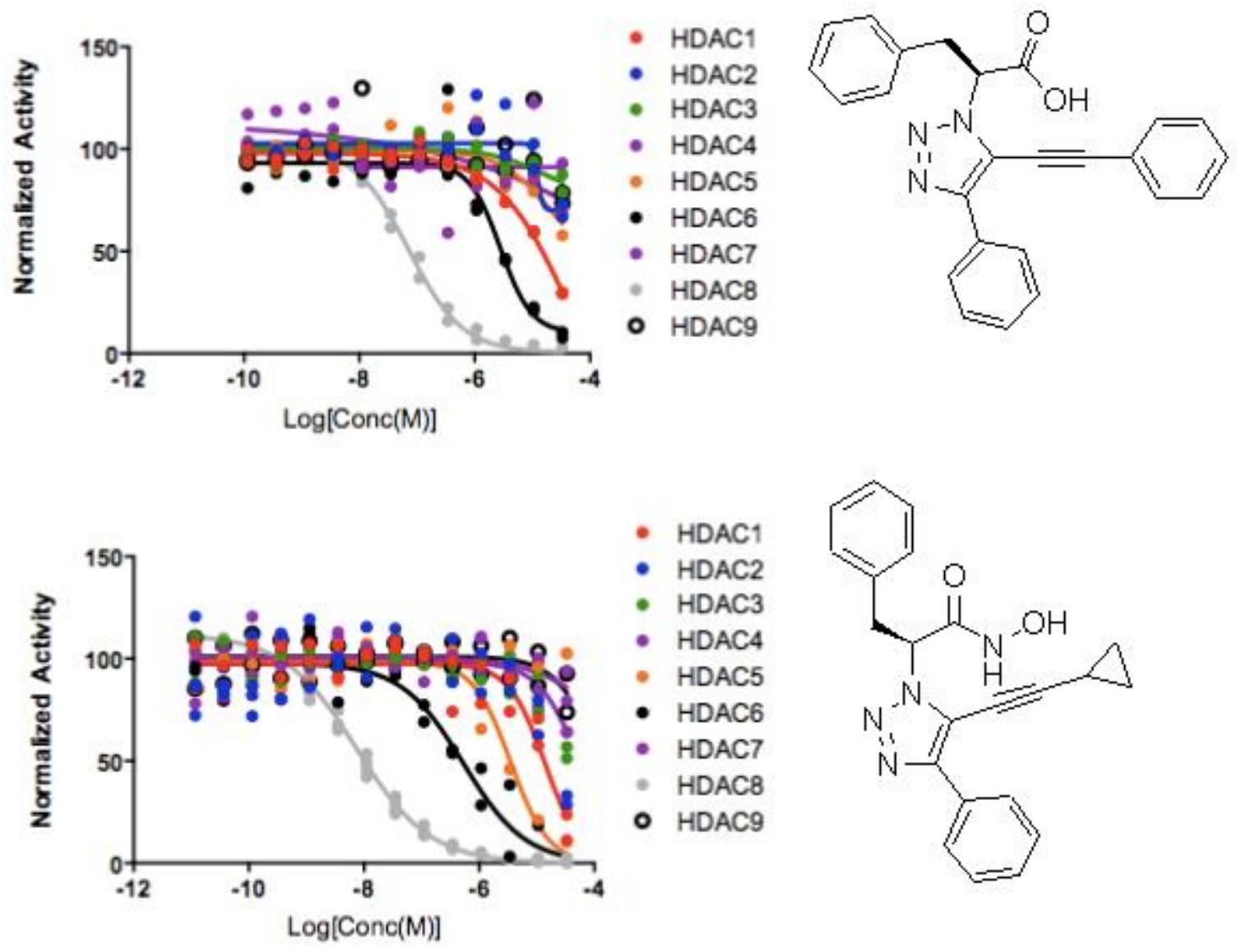

\footnotetext{
${ }^{2}$ Bowers, A.; West, N.; Taunton, J.; Schreiber, S. L.; Bradner, J. E.; Williams, R. M. J. Am. Chem. Soc 2008, 130,11219 ${ }^{3}$ Bowers, A.; Greshock, T.; West, N.; Estiu, G.;Schreiber, S.; Wiest, O.; Williams, R.; Bradner, J. J. Am. Chem. Soc. 2009, 131, 2900
} 


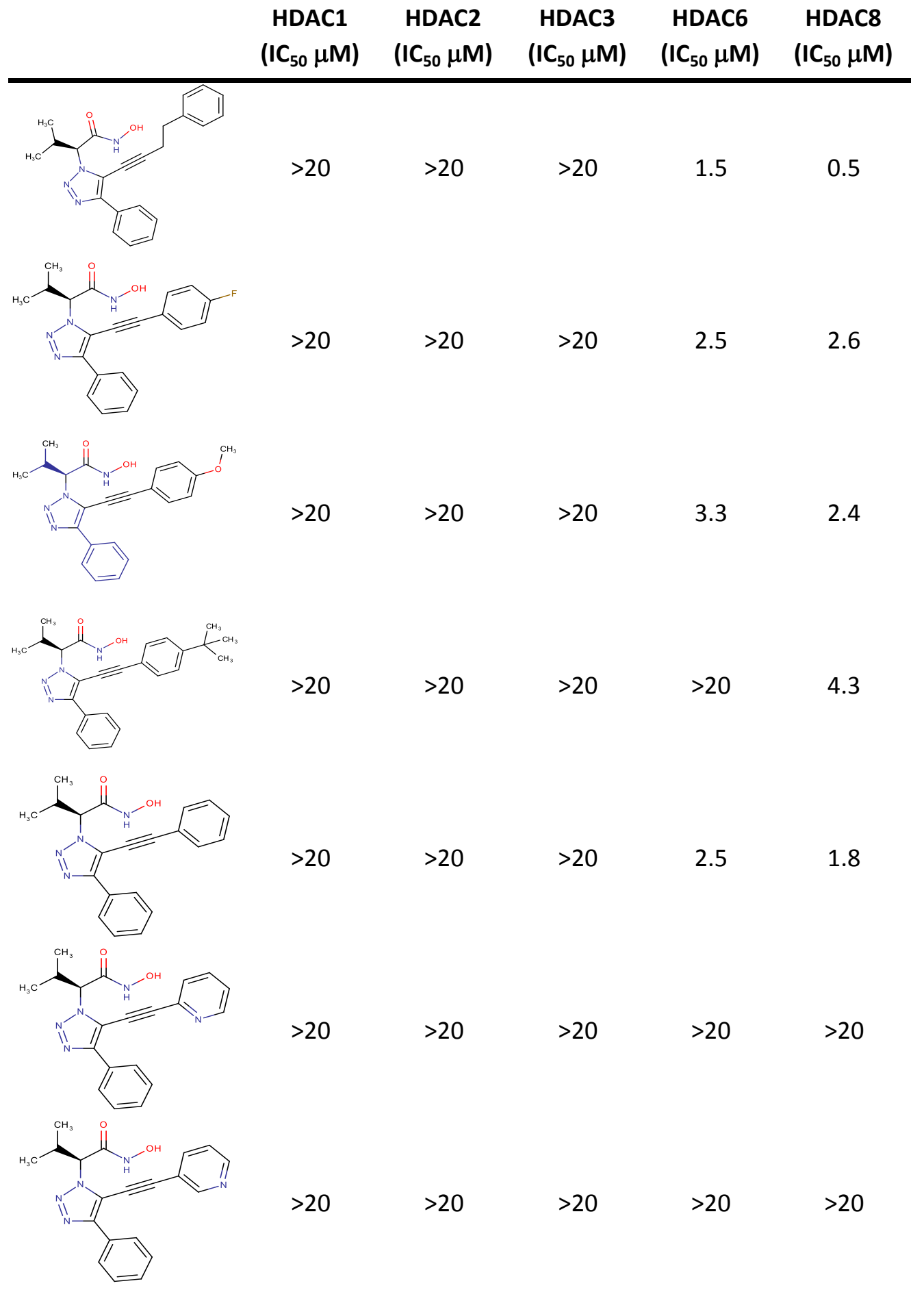




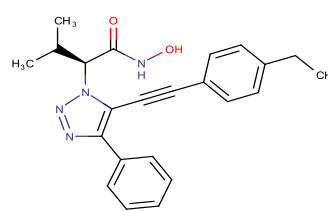

$>20$

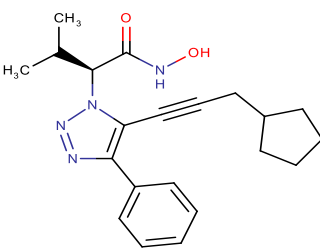

$>20$

$>20$

1

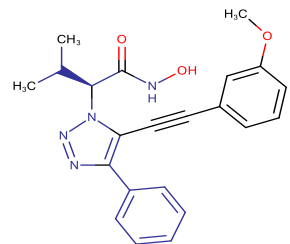

$>20$

$>20$

$>20$

$>20$

$>20$

2.6

4.5

3.2

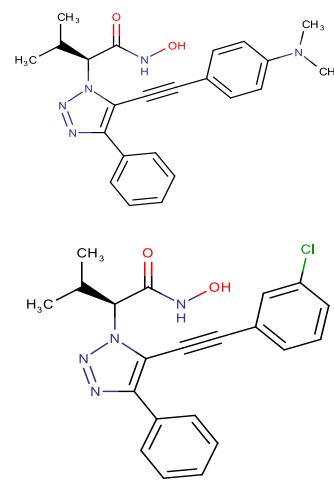

$>20$

$>20$

1.5

1.5

$>20$

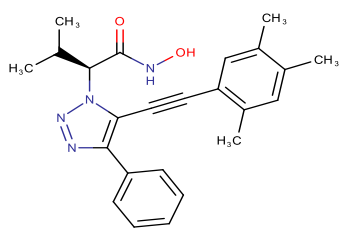

$>20$

$>20$

$>20$

NA

3.3

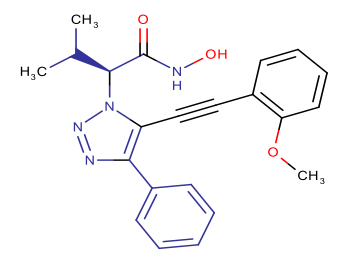

$>20$

$>20$

$>20$

3.4

3.1

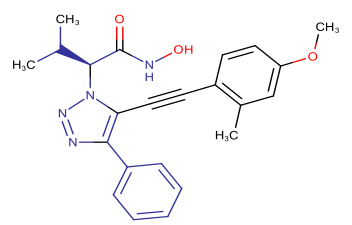


(1)

$>2$

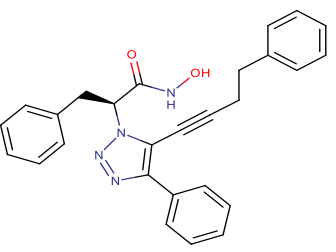

$>20$

17

$>20$

$>20$

$>20$

$>20$

$>20$

3.5

0.43

$>20$

$>20$

$>20$

10

5.5

$>20$

$>20$

$>20$

3.6

0.01

II

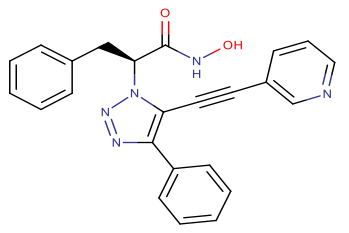

$>20$

$>20$

$>20$

7.5

0.15

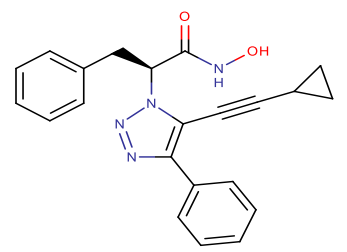

4.3

$>20$

$>20$

1.2

0.0008

$>20$

$>20$

$>20$

17

11 


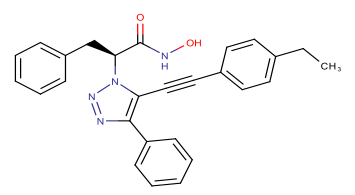

$>20$

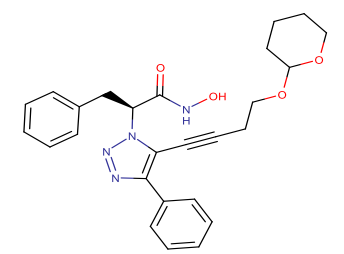

$>20$

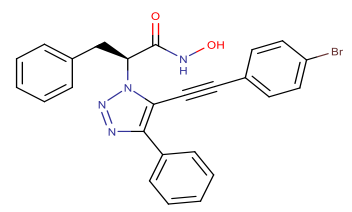

$>20$

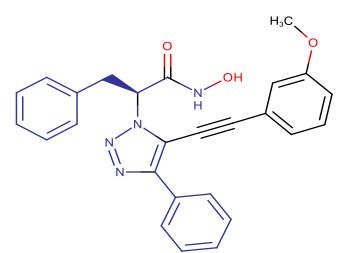

$>20$

$>20$

$>20$

$>20$

$>20$

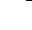

20

4.5

6.9



$>20$

$>20$

$>20$

1.4

2

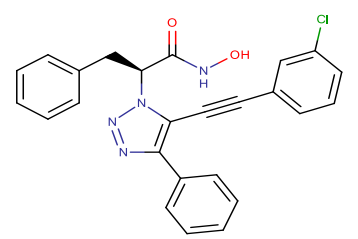

$>20$

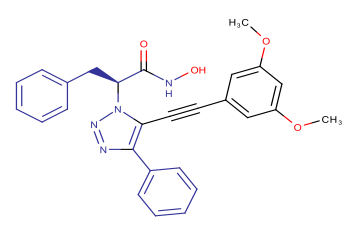

$>20$

$>20$

$>20$

$>20$

2.8

0.51



$>20$

$>20$

$>20$

12

2

0.28



$>20$

$>20$

4.5

0.31 


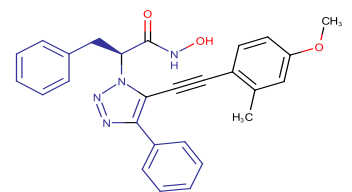

$>20$

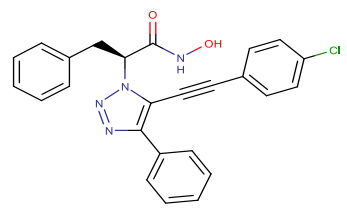

$>20$

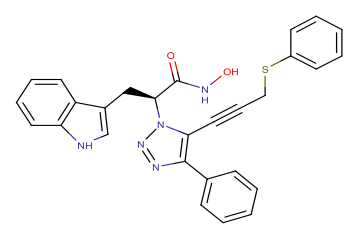

$>20$



$>20$


$>20$

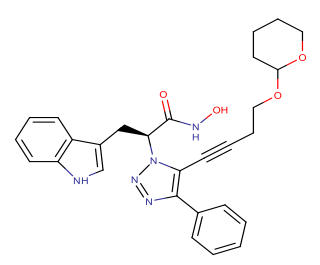

$>20$



$>20$

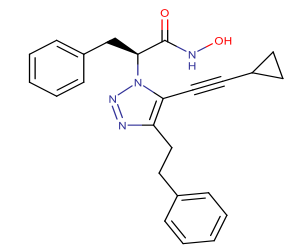

7.7

.7

NA
$>20$

$>20$

$>20$

$>20$

$>20$

$>20$

$>20$

$>20$

$>20$

1.2

0.0069

$>20$

$>20$

11.5

15.7

$>20$

$>20$

0.9

1.7

NA

$>20$

$>20$

2

0.33

NA

NA

0.353 


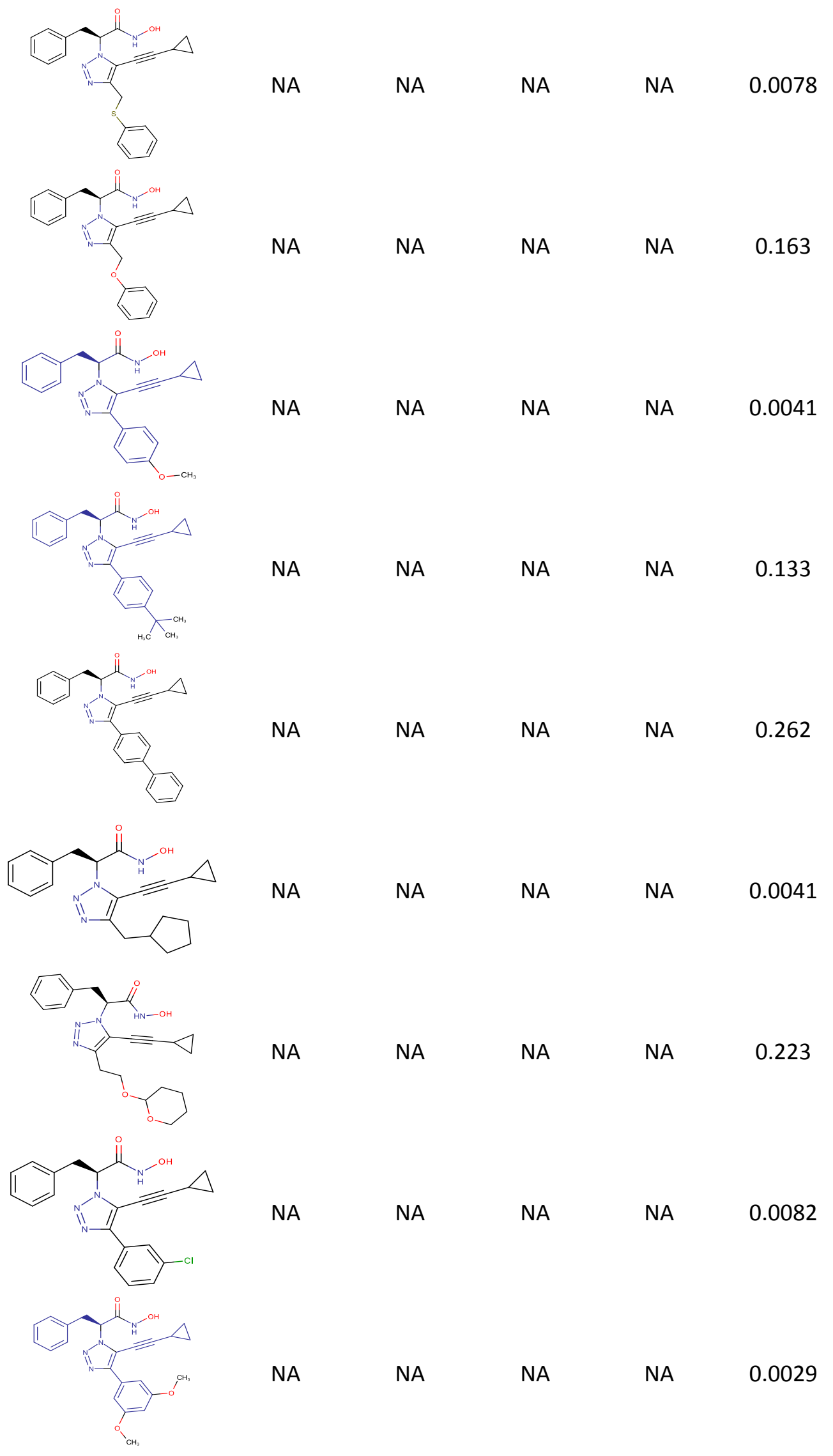




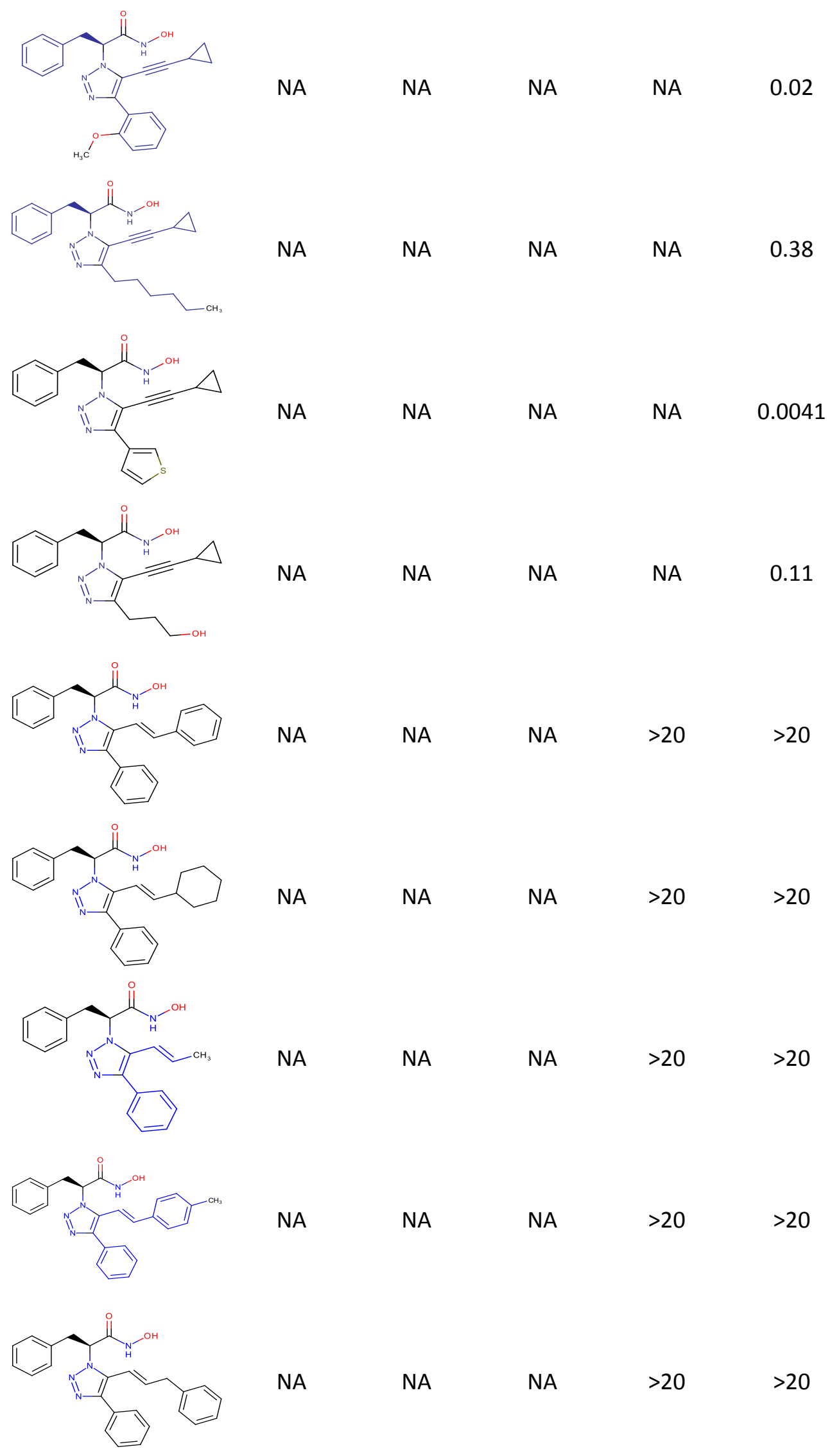






